\title{
Phosphorylation-independent activation of the atypical response regulator $\mathrm{NbIR}$
}

\section{Correspondence \\ Asunción Contreras \\ contrera@ua.es}

Received 20 May 2008

Revised 20 June 2008

Accepted 26 June 2008

\author{
Diego Ruiz, ${ }^{1}$ Paloma Salinas, ${ }^{1}$ Maria Luisa Lopez-Redondo, ${ }^{2}$ \\ Maria Luisa Cayuela, ${ }^{1}$ Alberto Marina ${ }^{2}$ and Asunción Contreras ${ }^{1}$ \\ ${ }^{1}$ División de Genética, Universidad de Alicante, Apartado 99, E-03080 Alicante, Spain
${ }^{2}$ Instituto de Biomedicina de Valencia (CSIC) and CIBERER, 46010 Valencia, Spain
}

Cyanobacteria respond to environmental stress conditions by adjusting their photosynthesis machinery. In Synechococcus sp. PCC 7942, phycobilisome degradation and other acclimation responses after nutrient or high-light stress require activation by the orphan response regulator NbIR, a member of the OmpR/PhoB family. Although NbIR contains a putative phosphorylatable residue (Asp57), it lacks other conserved residues required to chelate the $\mathrm{Mg}^{2+}$ necessary for aspartic acid phosphorylation or to transduce the phosphorylation signal. In close agreement with these features, NbIR was not phosphorylated in vitro by the low-molecular-mass phosphate donor acetyl phosphate and mutation of Asp57 to Ala had no impact on previously characterized NbIR functions in Synechococcus. On the other hand, in vitro and in vivo assays show that the default state of NbIR is monomeric, suggesting that, despite input differences, NbIR activation could involve the same general mechanism of activation by dimerization present in known members of the OmpR/PhoB family. Structural and functional data indicate that the receiver domain of NbIR shares similarities with other phosphorylation-independent response regulators such as FrzS and HP1043. To acknowledge the peculiarities of these atypical 'two-component' regulators with phosphorylation-independent signal transduction mechanisms, we propose the term PIARR, standing for phosphorylation-independent activation of response regulator.

\section{INTRODUCTION}

Cyanobacteria, photosynthetic prokaryotes that perform plant-type oxygenic photosynthesis, have developed mechanisms to modify the composition of their photosynthetic machinery in response to environmental changes (Grossman et al., 1993). One dramatic example of this adaptation is the process of chlorosis or bleaching, by which non-diazotrophic cyanobacteria degrade their lightharvesting antennae, the phycobilisomes, when exposed to stress conditions such as nutrient starvation (Collier \& Grossman, 1992). The small protein NblA (non-bleaching protein A) is required for phycobilisome degradation (Collier \& Grossman, 1994). The loss of phycobilisomes and a reduction of the chlorophyll content during stress conditions are responsible for the yellow appearance of chlorotic cultures. Degradation of phycobilisomes avoids excessive absorption of excitation energy and supplies the cell with amino acids for the synthesis of proteins required for acclimation and cell survival (Grossman et al., 2001).

Abbreviations: BACTH, bacterial adenylate cyclase two-hybrid; HK, histidine kinase; HL, high light; PIARR, phosphorylation-independent activation of response regulator; $\mathrm{RD}$, receiver domain; $\mathrm{RR}$, response regulator; RLU, relative light units.

A supplementary figure is available with the online version of this paper.
Two-component regulatory systems are widely used in signal transduction and adaptation to environmental changes in bacteria (Gao et al., 2007). In the prototype system a phosphate is transferred from a histidine in the dimerization and histidine phosphotransfer (DHp) domain of a sensor histidine kinase (HK) to an aspartate in the receiver domain $(\mathrm{RD})$ of a cognate response regulator (RR). Phosphorylation of the RD leads to conformational changes of the adjacent output domains. While the latter domain is structurally and functionally diverse, phosphorylation by cognate HKs requires structural conservation of RDs (reviewed in depth by Stock et al., 2000).

Consistent with their role in adaptation to environmental changes, a HK, NblS (van Waasbergen et al., 2002) and NblR (Schwarz \& Grossman, 1998), a RR from the OmpR/ PhoB subfamily, have been implicated in general adaptation to stress in Synechococcus sp. PCC 7942 (hereafter Synechococcus). NblS, an essential protein in Synechococcus, is the most conserved sensor $\mathrm{HK}$ in cyanobacteria, homologues being present in all cyanobacterial genomes available to date. Sequence analysis predicts the following domain organization for NblS: two N-terminal membranespanning regions (TM1 and TM2), a HAMP linker, a PAS domain and a conserved transmitter module, made up of a DHp domain and an ATP-binding domain (HATPase_c). 
Also conserved in cyanobacteria is SipA, a non-essential small regulatory factor that binds to the NblS HATPase domain and seems to cooperate with NblS in negative regulation of the $n b l A$ gene (Espinosa et al., 2006; Salinas et al., 2007). NblR is required for the strong increase in $n b l A$ gene expression observed during nutrient stress in Synechococcus (Luque et al., 2001; Salinas et al., 2007; Schwarz \& Grossman, 1998; Sendersky et al., 2005). The finding that point mutations in $n b l S$ also resulted in a nonbleaching phenotype (van Waasbergen et al., 2002) prompted suggestions of NblS being involved in the activation of NblR. However, NblR is required for induction of the chlorosis process, whereas the reported NblS mutant nblS-1, probably a gain-of-function mutant (Kappell et al., 2006), prevents chlorosis. NblR is also required for stress survival, and here NblS-SipA and NblR also seem to play opposite roles, as inferred from suppression of the $\mathrm{NblR}^{-}$phenotype by sipA inactivation (Salinas et al., 2007). Although the putative gene targets remain unknown, there are indications of a role for NblR in downregulation of photosynthetic electron transport under stress conditions (Schwarz \& Grossman, 1998). However, the mechanism and components involved in NblR activation remain to be elucidated.

In this work we provide and discuss structural and functional evidence indicating that activation of the NblR $\mathrm{RR}$ by stress signals does not rely on RD phosphorylation and it is therefore at odds with the paradigm for signal transduction by two-component RRs. To recognize the existence of alternative signal transduction mechanisms of atypical proteins whose RDs maintain most of the structural features of canonical RRs, but differ at residues directly involved in $\mathrm{RD}$ phosphorylation, we propose the term PIARR (phosphorylation-independent activation of response regulator).

\section{METHODS}

Molecular genetic techniques, culture conditions and twohybrid methods. Strains used in this work are listed in Table 1. Cloning procedures were carried out with Escherichia coli $\mathrm{DH} 5 \alpha$, using standard techniques.

Synechococcus strains were routinely grown photoautotrophically at $30{ }^{\circ} \mathrm{C}$ while shaking under constant illumination $(40 \mu \mathrm{mol}$ photons $\mathrm{m}^{-2} \mathrm{~s}^{-1}$ ) provided by cool white fluorescent lights. Media used were $\mathrm{BG}_{1} 1_{0}$ (no added nitrogen), BG11 (BG11 0 plus $17.5 \mathrm{mM} \mathrm{NaNO}_{3}$ and $10 \mathrm{mM}$ HEPES/NaOH pH 7.8) or BG11- $\mathrm{NH}_{4}\left(\mathrm{BG} 11_{0}\right.$ plus $10 \mathrm{mM}$ $\mathrm{NH}_{4} \mathrm{Cl}$ and $10 \mathrm{mM}$ HEPES/NaOH pH 7.8). For growth on plates, the medium was solidified by addition of $1 \%(\mathrm{w} / \mathrm{v})$ agar. Plates were incubated at $30{ }^{\circ} \mathrm{C}$ under constant illumination. Synechococcus strains were transformed as described by Golden \& Sherman (1984), incubated for $48 \mathrm{~h}$ at $30{ }^{\circ} \mathrm{C}$ under illumination on nitro-cellulose filters (Millipore), and transformants were selected on kanamycin-, chloramphenicol- or streptomycin-containing BG11 plates. For initiation of nitrogen deprivation, mid-exponential-phase BG11$\mathrm{NH}_{4}$ cultures grown with the appropriate antibiotics $\left(\mathrm{OD}_{750} 0.5\right)$ were harvested by centrifugation, washed twice with $\mathrm{BG} 11_{0}$ and finally resuspended in BG11 $1_{0}$ without antibiotics. For initiation of high-light (HL) stress, BG11 cultures were grown with the appropriate antibiotics to mid-exponential phase $\left(\mathrm{OD}_{750} 0.5\right)$ under standard illumination conditions, washed twice with BG11 and finally resuspended in BG11 without antibiotics and then transferred to HL conditions $\left(500 \mu \mathrm{mol}\right.$ photons $\left.\mathrm{m}^{-2} \mathrm{~s}^{-1}\right)$. To check the ability to resume growth, drops of the different strains previously subjected to stress were spotted at different times on BG11 plates without antibiotics. Antibiotic concentrations used for Synechococcus were $10 \mu \mathrm{g} \mathrm{ml}^{-1}$ (kanamycin) and $5 \mu \mathrm{g} \mathrm{ml} \mathrm{m}^{-1}$ (chloramphenicol and streptomycin).

Yeast culture and transformation procedures were as described by Ausubel et al. (1999). To perform yeast two-hybrid screenings, previously obtained Synechococcus Sau3AI (Burillo et al., 2004) or Tsp509I libraries (Burillo, 2006) were transformed into Saccharomyces cerevisiae PJ696 and mated to strain Y187 carrying either pUAGC3 or pUAGC301. To determine interaction patterns amongst selected proteins, expression from the three reporters present in PJ696/Y187 diploids was determined as previously described (Burillo et al., 2004).

For bacterial adenylate cyclase two-hybrid assays, E. coli DHM1 harbouring appropriate plasmid derivatives was grown at $25-28{ }^{\circ} \mathrm{C}$ with ampicillin $\left(50 \mu \mathrm{g} \mathrm{ml}^{-1}\right)$ and chloramphenicol $\left(30 \mu \mathrm{g} \mathrm{ml}^{-1}\right)$. Complementation was tested on M63 containing maltose $(0.3 \%)$, thiamin $(0.0001 \%)$, IPTG $(0.5 \mathrm{mM})$ and X-Gal $\left(80 \mu \mathrm{g} \mathrm{ml}^{-1}\right)$.

Modelling and structural alignment. The 3D structure model of the NblR receiver domain was produced by the combination of the structural models proposed by the Swiss-model repository (Kopp \& Schwede, 2004) and MODBASE (Pieper et al., 2004). This initial model was subsequently subjected to energy minimization using the program CNS (Brunger et al., 1998). The quality of the final model was assessed using the PROCHECK suite of programs (Morris et al., 1992). The receiver domain structures of phosphorylated Spo0A from Bacillus stearothermophilus (RCSB code 1QMP) (Lewis et al., 1999), beryllium fluoride-activated PhoB from E. coli (RCSB code 1ZES) (Bachhawat et al., 2005), FrzS from Myxococcus xanthus (RCSB code 2GKG) (Fraser et al., 2007), beryllium fluoride-activated CheY from E. coli (RCSB code 1FQW) (Lee et al., 2001), HP1043 from Helicobacter pylori (RCSB code 2PLN) as well as the NblR model were structurally aligned using the LSQKAB program as implemented in the CCP4 suite (Collaborative Computational Project, Number 4, 1994).

Construction of an $n b / R^{\mathrm{D} 57 \mathrm{~A}}$ mutant and derivative strains. An EcoRI-SalI fragment from plasmid pENS43 carrying the nblR gene was cloned into pBluescriptII SK( + ), giving plasmid pUAGC235. QuickChange Mutagenesis with primers NblR-D57A-F and NblRD57A-R and plasmid pUAGC235 as template resulted in plasmid pUAGC238, carrying $n b l R^{\mathrm{D} 57 \mathrm{~A}}$ (see Table 2 for primers). To generate appropriate flanking sites, the CS3 cassette $\left(\mathrm{Sm}^{\mathrm{R}}\right)$ from pRL453 was recloned into HindIII pBluescriptII $\mathrm{SK}(+)$, giving plasmid pUAGC453. A HincII-EcoRV fragment containing the CS3 cassette from pUAGC453 was then cloned into the Klenow-treated StyI site of pUAGC238 (downstream $n b l R^{\mathrm{D} 57 \mathrm{~A}}$ ), giving plasmid pUAGC240. Downstream sequences of $n b l R(670 \mathrm{bp})$ were amplified from genomic Synechococcus DNA using primers NblR-down-4F and NblR-down-4R. The PCR product was then cut with HindIII and HincII and Klenow filled. This blunt fragment was then cloned into HincII-cut pUAGC240, giving plasmid pUAGC239, carrying the CS3 cassette between nblR and the ORF Synpcc7942_2306. Transformation of pUAGC239 into Synechococcus resulted in stable chromosomal integration of the CS3 cassette and adjacent sequences, as confirmed by PCR with primers NbIR-1F and CS3-2R. The presence of $n b l R^{\mathrm{D} 57}$ or $n b l R$ alleles was checked by a second PCR with primers NblR-1F and NblR-1R followed by digestion with PvuI, giving 309, 186 and $51 \mathrm{bp}$ fragments for wild-type $n b l R$ and 309 and $237 \mathrm{bp}$ fragments for $n b l R^{\mathrm{D} 57 \mathrm{~A}}$. A clone of each type was selected for further analysis (strains WT-RCS3 and $\mathrm{NblR}^{\mathrm{D} 57 \mathrm{~A}}-\mathrm{RCS} 3$, respectively). 
Table 1. Strains and plasmids

\begin{tabular}{|c|c|c|}
\hline Strain or plasmid & Genotype or relevant characteristics & Source or reference \\
\hline E. coli DH5 $\alpha$ & $\begin{array}{l}\mathrm{F}^{-} \phi 80 \mathrm{~d} l a c Z \Delta \mathrm{M} 15 \Delta(\text { lacZYA-argF }) U 169 \text { endA1 recA1 hsdR17 }\left(\mathrm{r}_{\mathrm{K}}^{-} \mathrm{m}_{\mathrm{K}}^{+}\right) \\
\text {deoR thi-1 supE44 gyrA96 relA1 } \lambda^{-}\end{array}$ & Hanahan (1985) \\
\hline E. coli DHM1 & $\mathrm{F}^{-}$glnV44(AS) recA1 endA gyrA46 thi-1 hsdR17 spoT1 frbD1 cya-854 & Karimova et al. (2005) \\
\hline E. coli BL21-CodonPlus(DE3)-RIL & $\begin{array}{l}\text { E. coli B F }{ }^{-} \text {ompT hsdS }\left(\mathrm{r}_{\mathrm{B}}^{-} \mathrm{m}_{\mathrm{B}}^{-}\right) \mathrm{dcm}^{+} \operatorname{Tet}^{\mathrm{r}} \text { gal } \lambda(\mathrm{DE} 3) \text { endA Hte } \\
{\left[\arg U \text { ileY leuW } \mathrm{Cam}^{\mathrm{r}}\right]}\end{array}$ & Stratagene \\
\hline S. cerevisiae Y187 & 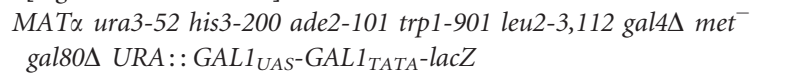 & Harper et al. (1993) \\
\hline S. cerevisiae PJ696 & 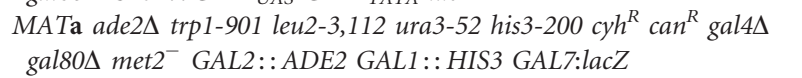 & James et al. (1996) \\
\hline Synechococcus sp. PCC 7942 & Wild-type Synechococcus sp. PCC 7942 & Pasteur culture collection \\
\hline Synechococcus NblR45 & $\mathrm{NblR}^{-}, \mathrm{Km}^{\mathrm{r}}$ & Luque et al. (2001) \\
\hline Synechococcus WT-C103 & $\mathrm{P}_{n b l A}:: \operatorname{lux} A B$ into NSII, $\mathrm{Cm}^{\mathrm{r}}$ & Espinosa et al. (2007) \\
\hline Synechococcus NblR45-C103 & $\mathrm{NblR}^{-}, \mathrm{P}_{n b l A}:: \operatorname{lux} A B, \mathrm{Cm}^{\mathrm{r}} \mathrm{Km}^{\mathrm{r}}$ & Salinas et al. (2007) \\
\hline Synechococcus WT-RCS3 & CS3 downstream $n b l R, \mathrm{Sm}^{\mathrm{r}}$ & This work \\
\hline Synechococcus NblR ${ }^{\mathrm{D} 57 \mathrm{~A}}-\mathrm{RCS} 3$ & $\mathrm{NblR}^{\mathrm{D} 57 \mathrm{~A}}, \mathrm{Sm}^{\mathrm{r}}$ & This work \\
\hline Synechococcus WT-RCS3-C103 & $\mathrm{P}_{n b l A}:: \operatorname{lux} A B, \mathrm{Cm}^{\mathrm{r}} \mathrm{Sm}^{\mathrm{r}}$ & This work \\
\hline $\begin{array}{l}\text { Synechococcus } \text { NblR }^{\text {D57A }} \\
\text { RCS3-C103 }\end{array}$ & $\mathrm{NblR}^{\mathrm{D} 57 \mathrm{~A}}, \mathrm{P}_{n b l A}:: \operatorname{lu} x A B, \mathrm{Cm}^{\mathrm{r}} \mathrm{Sm}^{\mathrm{r}}$ & This work \\
\hline pBluescriptII SK(+) & Cloning vector, $A p^{r}$ & Stratagene \\
\hline pENS43 & $1.8 \mathrm{~kb}$ genomic $n b l R$ fragment cloned into pRL519, $\mathrm{Ap}^{\mathrm{r}}$ & Luque et al. (2001) \\
\hline pENS38 & pPROEX-HTb encoding 6His-NblR, $\mathrm{Ap}^{\mathrm{r}}$ & Luque et al. (2001) \\
\hline pNblR-RD & 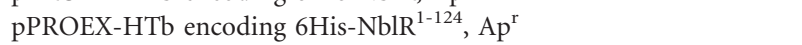 & This work \\
\hline pTM0468 & pET22b encoding full-length RR468, $\mathrm{Ap}^{\mathrm{r}}$ & Casino et al. (2007) \\
\hline pUAGC235 & pBluescriptII SK( $(+)$ with $n b l R, \mathrm{Ap}^{\mathrm{r}}$ & This work \\
\hline pUAGC238 & pBluescriptII SK $(+)$ with $n b l R^{\mathrm{D} 57 \mathrm{~A}}, \mathrm{Ap}^{\mathrm{r}}$ & This work \\
\hline pUAGC240 & pUAGC238 with CS3, Ap ${ }^{\mathrm{r}} \mathrm{Sm}^{\mathrm{r}}$ & This work \\
\hline pUAGC239 & pUAGC240 with $n b l R$ downstream sequences, $\mathrm{Ap}^{\mathrm{r}} \mathrm{Sm}^{\mathrm{r}}$ & This work \\
\hline pUAGC453 & pBluescriptII SK $(+)$ with CS3, $A^{\mathrm{r}}{ }^{\mathrm{S}} \mathrm{s}^{\mathrm{r}}$ & This work \\
\hline pGAD424 & LEU2, GAL4(768-881)AD, Ap $^{\mathrm{r}}$ & Bartel et al. (1993) \\
\hline pGBT9 & TRP1, GAL4(1-147)BD, Ap ${ }^{\mathrm{r}}$ & Bartel et al. (1993) \\
\hline pGAD $424(+1)$ & As pGAD424 with a different frame $(+1), \mathrm{Ap}^{\mathrm{r}}$ & Roder et al. (1996) \\
\hline $\operatorname{pGBT9}(+1)$ & As pGBT9 with a different frame $(+1)$, Ap $^{\mathrm{r}}$ & Roder et al. (1996) \\
\hline pADC & LEU2, GAL4-AD for C-terminal cloning, Ap ${ }^{\mathrm{r}}$ & Millson et al. (2003) \\
\hline pBDC & TRP1, GAL4-BD for C-terminal cloning, $\mathrm{Ap}^{\mathrm{r}}$ & Millson et al. (2003) \\
\hline pUAGC51 & GAL4AD : NblS ${ }^{272-664}, \mathrm{Ap}^{\mathrm{r}}$ & Espinosa et al. (2006) \\
\hline pUAGC52 & GAL4BD : NblS ${ }^{272-664}, \mathrm{Ap}^{\mathrm{r}}$ & Espinosa et al. (2006) \\
\hline pUAGC53 & GAL4AD : NblS, $\mathrm{Ap}^{\mathrm{r}}$ & Espinosa et al. (2006) \\
\hline pUAGC54 & GAL4BD : NblS, $\mathrm{Ap}^{\mathrm{r}}$ & Espinosa et al. (2006) \\
\hline pUAGC1 & GAL4AD: NblR, $\mathrm{Ap}^{\mathrm{r}}$ & Burillo (2006) \\
\hline pUAGC3 & GAL4BD : NblR, Ap ${ }^{\mathrm{r}}$ & Burillo (2006) \\
\hline pUAGC300 & GAL4AD : $\mathrm{NblR}^{1-171}, \mathrm{Ap}^{\mathrm{r}}$ & This work \\
\hline pUAGC301 & GAL4BD : $\mathrm{NblR}^{1-171}, \mathrm{Ap}^{\mathrm{r}}$ & This work \\
\hline pUAGC597 & NblR: GAL4AD, Ap ${ }^{\mathrm{r}}$ & This work \\
\hline pUAGC598 & NblR: GAL4BD, $A p^{r}$ & This work \\
\hline pUAGC615 & GAL4AD: NarB, $\mathrm{Ap}^{\mathrm{r}}$ & This work \\
\hline pUAGC616 & GAL4BD : NarB, $\mathrm{Ap}^{\mathrm{r}}$ & This work \\
\hline pUAGC310 & GAL4BD : $\mathrm{NarB}^{154-344}, \mathrm{Ap}^{\mathrm{r}}$ & This work \\
\hline pUAGC311 & GAL4AD : NarB ${ }^{154-344}, \mathrm{Ap}^{\mathrm{r}}$ & This work \\
\hline pUAGC312 & GAL4BD : NarB ${ }^{154-255}, \mathrm{Ap}^{\mathrm{r}}$ & This work \\
\hline pUAGC313 & GAL4AD : $\operatorname{NarB}^{154-255}, \mathrm{Ap}^{\mathrm{r}}$ & This work \\
\hline pUAGC314 & GAL4BD : NarB ${ }^{112-382}, \mathrm{Ap}^{\mathrm{r}}$ & This work \\
\hline pUAGC315 & GAL4AD : $\mathrm{NarB}^{112-382}, \mathrm{Ap}^{\mathrm{r}}$ & This work \\
\hline pUAGC324 & GAL4BD: $\mathrm{NdhH}^{54-283}, \mathrm{Ap}^{\mathrm{r}}$ & This work \\
\hline pUAGC325 & GAL4AD : $\mathrm{NdhH}^{54-283}, \mathrm{Ap}^{\mathrm{r}}$ & This work \\
\hline pT25 & CyaA $(1-224) \mathrm{T} 25, \mathrm{Cm}^{\mathrm{r}}$ & Karimova et al. (1998) \\
\hline pUT18c & CyaA(225-399)T18, Ap ${ }^{\mathrm{r}}$ & Karimova et al. (1998) \\
\hline pUAGC439 & $\mathrm{T} 25: \mathrm{NblS}, \mathrm{Cm}^{\mathrm{r}}$ & Espinosa et al. (2006) \\
\hline pUAGC600 & $\mathrm{T} 25: \mathrm{NblR}, \mathrm{Cm}^{\mathrm{r}}$ & This work \\
\hline pUAGC601 & $\mathrm{T} 18: \mathrm{NblR}, \mathrm{Ap}^{\mathrm{r}}$ & This work \\
\hline pUAGC604 & $\mathrm{T} 25: \mathrm{NblR}^{125-229}, \mathrm{Cm}^{\mathrm{r}}$ & This work \\
\hline pUAGC605 & $\mathrm{T} 18: \mathrm{NblR}^{125-229}, \mathrm{Ap}^{\mathrm{r}}$ & This work \\
\hline pUAGC602 & $\mathrm{T} 25: \mathrm{NarB}, \mathrm{Cm}^{\mathrm{r}}$ & This work \\
\hline pUAGC603 & T18:NarB, Ap ${ }^{r}$ & This work \\
\hline
\end{tabular}


Table 2. Oligonucleotides

\begin{tabular}{|c|c|}
\hline Name & Sequence \\
\hline ADC-F & 5'-CAAGCTATACCAAGCATACAATCCAAGATG-3' \\
\hline ADC-R & 5'-GGAATTAATTCCGCTTTATCCATCTTTGCAAAGGC-3' \\
\hline BDC-F & 5'-CAACTCCAAGCTTGAAGCAAGCCTCGATG-3' \\
\hline BDC-R & 5'-CGATAGAAGACAGTAGCTTCATCTTTCG-3' \\
\hline CS3-2R & 5'-ACAAAACGGTTTACCAGCAT-3' \\
\hline NblR-1F & 5'-GAGTGAGGAAGAATTCTGATCGCGCCAGCCTC-3' \\
\hline NblR-1R & 5'-CTCTCGCGTCGACACTTAGCGCGGATGCTC-3' \\
\hline NblR-3 & 5'-GGCAGCGCAGTCGACTTTGAAGCCATG-3' \\
\hline NarB-1F & 5'-GAGGGAATTCTCGATCTCGATCTCTCGAAGTTTC-3' \\
\hline NarB-1R & 5'-GCCTAGAGGTCGACTTAGCGATCG-3 \\
\hline NblR ADC-F & 5'-AGCATACAATCCAAGATGATCGCGCCAGCCTCGCCACACA-3' \\
\hline NblR ADC-R & 5'-CGCTTTATCCATCTTTGCAAAGGCGCTTTCGCGTAGGACATAACCG-3' \\
\hline NblR BDC-F & 5'-GCTTGAAGCAAGCCTCGATGATCGCGCCAGCCTCGCCACAC-3' \\
\hline NblR BDC-R & 5'-CAGTAGCTTCATCTTTCGGCTTTCGCGTAGGACATAACC-3' \\
\hline Transgadgbt-1F & $\begin{array}{l}\text { 5'-CGCACATCATCATCGGAAGAGAGTAGTAACAAAGGTCAAAGAC } \\
\text { AGTTGACTGTATCGCCGAACCCAAAAAAAGAGATCG-3' }\end{array}$ \\
\hline Transgadgbt-1R & $\begin{array}{l}\text { 5'-ATAACTTATTTAATAATAAAAATCATAAATCATAAGAAATTCGCCCGGAATT } \\
\text { AGCTTGGCGTTTTTCAGTATCTACGATTC-3' }\end{array}$ \\
\hline NblR bth1-F & 5'-TGAGGGGATCCTATGATCGCGC-3' \\
\hline NblR bth1-R & 5'-ATCGCGGTACCTTAGCTTTCGC-3' \\
\hline NblR bth2-F & 5' TACAGGATCCACCTGCTGCTCA-3' \\
\hline NarB bth-1F & 5'-GCGCGGATCCAATGTTCGATC-3' \\
\hline NarB bth-1R & 5'-TTGCGGTACCTTAGATTGTGAAG-3' \\
\hline NblR-D57A-F & 5'-GATTTAGTCATCGTCGCTCGCATGGCGGGCGG-3' \\
\hline NblR-D57A-R & 5'-CCGCCCGCCATGCGAGCGACGATGACTAAATC-3' \\
\hline NblR-down-4F & 5'-TGTAGAAGCTTGCGGCTC-3' \\
\hline NblR-down-4R & 5'-CTTTAGGTCGACTGCAGTCTCGTCATC-3' \\
\hline NblR-RD-F & 5'-GGCGCCATGGTCGCGCCAGCCTCGCC-3' \\
\hline NblR-RD-R & 5'-GCAGAAGCTTATCGTTGTAAATAGAG-3' \\
\hline
\end{tabular}

To obtain wild-type and $n b l R^{\mathrm{D} 57}$ derivatives carrying the $\mathrm{P}_{n b l A}::$ luxAB reporter fusion, plasmid pUAGC239 was transformed into strain WT-C103 (Espinosa et al., 2007) and transformant clones analysed as above to independently recover each type of $n b l R$ alleles (strains WT-RCS3-C103 and NblR ${ }^{\text {D57A }}$-RCS3-C103).

Construction of yeast and bacterial two-hybrid plasmids. A 485 bp fragment from the $n b l R$ gene was PCR amplified with primers NblR-1F and NblR-1R, cut with EcoRI and SalI and cloned into $\operatorname{pGAD} 424(+1)$ and $\operatorname{pGBT9}(+1)$, giving plasmids pUAGC300 $\left(\right.$ GAL4AD: $\mathrm{NblR}^{1-171}$ ) and pUAGC301 (GAL4BD: NblR ${ }^{1-17}$ ), respectively. To produce a NblR:GAL4AD protein fusion, PCR amplification of $n b l R$ sequences with primers NblR ADC-F and NblR ADC-R was followed by a second PCR with primers ADC-F and ADC-R. This product was mixed with NruI-opened pADC and transformed into S. cerevisiae PJ696, to produce, by homologous recombination, plasmid pUAGC597. Similarly, to obtain a NblR: GAL4BD protein fusion, primers NblR BDC-F and NblR BDC-R and then BDC-F and BDC-R were used in consecutive PCRs, the product mixed with linear $\mathrm{pBDC}$ and transformed into $S$. cerevisiae Y187, giving plasmid pUAGC598. To obtain bacterial adenylate cyclase two-hybrid (BACTH) derivatives, nblR was PCR amplified with primers NblR bth1-F and NblR bth1-R, cut with BamHI and KpnI, and cloned into pT25 or pUT18c, resulting in plasmids pUAGC600 (T25:NblR) and pUAGC601 (T18:NblR), respectively. The same procedure but using primers NblR bth2-F and NblR bth1-R resulted in plasmids pUAGC604 (T25: NblR ${ }^{125-229}$ ) and pUAGC605 (T18: NblR $\left.^{125-229}\right)$.
The narB sequence was PCR amplified using primers NarB bthl-F and NarB bth1-R, cut with BamHI and KpnI, and cloned into pT25 or pUT18c, resulting in plasmids pUAGC602 (T25:NarB) and pUAGC603 (T18:NarB), respectively. To obtain plasmids pUAGC615 (GAL4AD: NarB) and pUAGC616 (GAL4BD: NarB), the narB sequence was amplified with primers NarB-1F and NarB-1R, cut with EcoRI and Sall and cloned into pGAD424 $(+1)$ and pGBT9 $(+1)$.

Inserts from prey plasmids pUAGC311 (GAL4AD: $\mathrm{NarB}^{154-344}$ ), pUAGC313 (GAL4AD:NarB ${ }^{154-255}$ ) and pUAGC315 (GAL4AD: $\mathrm{NarB}^{112-382}$, obtained in yeast two-hybrid screenings using NblR as bait, were PCR amplified using primers transgadgbt-1F and transgadgbt-1R. The PCR together with linearized (EcoRI-SalI) pGBT9 was then transformed into S. cerevisiae PJ696, giving plasmids pUAGC310 (GAL4BD: $\mathrm{NarB}^{154-344}$ ), pUAGC312 (GAL4BD: $\mathrm{NarB}^{154-255}$ ) and pUAGC314 (GAL4BD: NarB $^{112-382}$ ). The same strategy was used to obtain plasmid pUAGC324 (GAL4BD : $\mathrm{NdhH}^{54-283}$ ) from pUAGC325 (GAL4AD : $\mathrm{NdhH}^{54-283}$ ).

Protein cloning, expression and purification. DNA sequences encoding residues 1-124 of NblR (6His-NblR-RD) were PCR amplified from Synechococcus genomic DNA using primers NblRRD-F and NblR-RD-R. The PCR product was cloned into the NcoI and HindIII sites of plasmid pPROEX-HTb (Invitrogen), creating an in-frame fusion to the $\mathrm{N}$-terminal $\mathrm{His}_{6}$-tag. The resulting plasmid was named pNblR-RD.

The RR468 protein was expressed and purified as previously described (Casino et al., 2007). For expression of 6His-NblR and 6His-NblR-RD 
proteins, plasmids pENS38 and pNblR-RD were transformed into the E. coli strain BL21-codonPlus (DE3)-RIL (Stratagene). Cells were grown in the autoinductive ZYP-5052 medium supplemented with ampicillin $\left(100 \mu \mathrm{g} \mathrm{ml}^{-1}\right)$ and chloramphenicol $\left(33 \mu \mathrm{g} \mathrm{ml}^{-1}\right)$, and the expression of proteins was carried out following an autoinduction method (Studier, 2005). Cells were harvested, disrupted by sonication, and the soluble fraction was purified by Ni-affinity chromatography using Protino Ni-TED 1000 (Macherey-Nagel). Elution of the protein was carried out using $250 \mathrm{mM}$ imidazol. The purest fractions (as determined by SDS-PAGE and Coomassie blue staining) were pooled, washed with storage buffer $(25 \mathrm{mM}$ Tris/ $\mathrm{HCl} \mathrm{pH} 8.0$, $100 \mathrm{mM} \mathrm{NaCl}$ ), concentrated using Amicon Ultra (Millipore) and stored at $-80{ }^{\circ} \mathrm{C}$. Proteins were quantified spectrophotometrically using the method of Bradford (Bio-Rad).

Phosphorylation assay with acetyl phosphate and resolution by native PAGE and 2D gel electrophoresis. Purified $6 \mathrm{His}-\mathrm{NblR}$, 6His-NblR-RD and RR468 were autophosphorylated in kinase buffer (50 mM Tris $\mathrm{pH} 8.0,100 \mathrm{mM} \mathrm{KCl}, 10 \mathrm{mM} \mathrm{MgCl}_{2}$ ) containing $12.5 \mathrm{mM}$ acetyl phosphate for $1 \mathrm{~h}$ at room temperature. After phosphorylation, loading buffer (62.5 mM Tris pH 6.8, $30 \%$ glycerol, $0.01 \%$ bromophenol blue) was added in a $1: 4$ ratio to the samples and these were subsequently subjected to Native PAGE on a $10 \%$ gel at $100 \mathrm{~V}$ for $2 \mathrm{~h}$ at $4{ }^{\circ} \mathrm{C}$.

To evaluate phosphorylation by $2 \mathrm{D}$ gel electrophoresis, the reactions with acetyl phosphate were carried out in a final volume of $10 \mu \mathrm{l}$, including $15 \mu \mathrm{g} 6$ His-NblR-RD or $6 \mu \mathrm{g}$ RR468. The phosphorylation reactions were stopped by the addition of $90 \mu \mathrm{l}$ lysis buffer ( $8 \mathrm{M}$ urea, $2 \%$ CHAPS), $1 \mu \mathrm{l} 1 \mathrm{M}$ DTT and $0.5 \mu \mathrm{l}$ ampholytes $\mathrm{pH}$ 4-7. Control samples without acetyl phosphate were run in parallel. Samples consisting of the unphosphorylated proteins or a mix of equal amounts of unphosphorylated and phosphorylated proteins, where the unphosphorylated proteins were used as internal control, were isoelectrically focused for each protein in the first dimension using a $7 \mathrm{~cm}$ Immobiline Drystrip (pH 4.0-7.0; GE Healthcare) and an Ettan IPGphor (Amershan Biosciences) system following the indications of the manufacturers. The samples were focused at $500 \mathrm{~V}$ for $0.25 \mathrm{kV} \mathrm{h}$, $1000 \mathrm{~V}$ for $0.5 \mathrm{kV}$ h and $6000 \mathrm{~V}$ for $7.5 \mathrm{kV}$ h at $20{ }^{\circ} \mathrm{C}$. Proteins were resolved in the second dimension by SDS-PAGE using $15 \%$ acrylamide gels.

Both types of gels were stained with Coomassie blue and images analysed using a Fuji LAS-3000 imaging system and the MultiGauge Fujifilm program.

Gel filtration chromatography. Gel filtration chromatography was carried out in a Superdex 200 HR 10/30 column (Amersham Biosciences) equilibrated with running buffer ( $50 \mathrm{mM}$ Tris $\mathrm{pH} 7.5$, $150 \mathrm{mM} \mathrm{NaCl}$ ) and calibrated with a cocktail of molecular mass standards, containing Blue Dextran 2000 ( 2000 kDa), $\beta$-amylase $(200 \mathrm{kDa})$, alcohol dehydrogenase $(150 \mathrm{kDa})$, BSA $(66 \mathrm{kDa})$, carbonic anhydrase $(29 \mathrm{kDa})$, cytochrome $c(12.4 \mathrm{kDa})$ and ATP $(0.57 \mathrm{kDa})$. Samples $(100 \mu \mathrm{l})$ of $6 \mathrm{His}-\mathrm{NblR}$ or 6 His-NblR-RD containing $150 \mu \mathrm{g}$ protein in running buffer were individually applied to the column, eluted with running buffer at a flow rate of $0.5 \mathrm{ml}$ $\min ^{-1}$ and collected in $1 \mathrm{ml}$ fractions. The fractions were analysed by SDS-PAGE. Protein elution profiles were monitored by measuring the absorbance at $280 \mathrm{~nm}$. For evaluation of the acetyl phosphate effect, the samples were incubated for $1 \mathrm{~h}$ at room temperature with acetyl phosphate and $\mathrm{MgCl}_{2}(12.5 \mathrm{mM})$ before chromatography. Sample oxidation was carried out by treatment with $0.3 \mathrm{mM} \mathrm{Cu}(\mathrm{II})-(o-$ phenanthroline $)_{3}$ (Lee et al., 1995) for $30 \mathrm{~min}$ at $37^{\circ} \mathrm{C}$ prior to gel filtration. Sample reduction was carried out by incubation with $10 \mathrm{mM}$ DTT for $30 \mathrm{~min}$ at room temperature, and the chromatography running buffer was supplemented with $1 \mathrm{mM}$ DTT.
Determination of luciferase activity. To determine bioluminescence, $1 \mathrm{ml}$ of cultures grown to mid-exponential phase were adjusted to an $\mathrm{OD}_{750}$ of 0.5 and supplemented with decanal to a final concentration of $0.25 \mathrm{mM}$ from a $50 \mathrm{mM}$ stock solution made up in $10 \%$ DMSO. Light emission was recorded in a Berthold LB9509 luminometer. Bioluminescence was recorded every $20 \mathrm{~s}$ for $10 \mathrm{~min}$. Light emission increased to a maximum and then declined. Maximum luminescence at the peak, presented as RLU (relative light units) by the instrument, is the value used at each selected time point.

Determination of pigment contents spectrophotometrically. Whole-cell absorbance spectra were obtained in order to estimate pigment contents. Samples $(1 \mathrm{ml})$ of cultures were taken at the indicated times, diluted with fresh medium to an $\mathrm{OD}_{750} 0.5$, and absorbance spectra $(500-800 \mathrm{~nm})$ were recorded on a UV/Visible Ultrospec 2100 pro (GE Healthcare Life Sciences). Pigment content was calculated based on absorbance maxima at $631 \mathrm{~nm}$ for phycocyanin and $684 \mathrm{~nm}$ for chlorophyll $a$, essentially as described by Myers et al. (1980).

\section{RESULTS AND DISCUSSION}

\section{NbIR lacks residues conserved in canonical response regulators}

Three groups of amino acids are essential for phosphorylation of RDs and the consequent response: the phosphoaccepting aspartate (Asp57 in CheY numeration), the catalytic residues (Asp12, Asp13 and Lys109) and the conformational switch residues (Thr87 and Tyr106) (Dyer \& Dahlquist, 2006). However, NblR lacks two of these key amino acids (see Fig. 1b). In this context, it is worth noting recent reports of atypical RRs with striking deviations from canonical RDs (Fraser et al., 2007; Schar et al., 2005). To illustrate key discrepancies between canonical RDs, NblR $\mathrm{RD}$ and other atypical $\mathrm{RDs}$, we produced a $3 \mathrm{D}$ structure model of the NblR RD and used it to generate a structural alignment with selected canonical and non-canonical RDs. In order to emphasize the catalytic residue disposition in the alignment, we chose the $\mathrm{CheY}$ and PhoB beryllium fluoride-activated structures (Lee et al., 2001) as prototypical RDs, as well as the phosphorylated Spo0A structure (Lewis et al., 1999), the first canonical RD crystallized in the phosphorylated form. The structures of Myxococcus xanthus FrzS (Fraser et al., 2007) and Helicobacter pylori HP1043 (Schar et al., 2005) were used as a model of atypical RDs. As shown in Fig. 1(a), NblR apparently retains the overall folding of RDs, as is the case with the RDs from FrzS and HP1043. In NblR, the highly conserved aspartic acid and threonine residues (Asp13 and Thr87 in CheY) are substituted by serine and methionine (Ser14 and Met85), respectively. Asp13, probably the main residue involved in $\mathrm{Mg}^{2+}$ binding (Hubbard et al., 2003; Lee et al., 2001), is substituted for Ser and Lys, respectively, in FrzS and HP1043. Consistent with these changes, FrzS is insensitive to $\mathrm{Mg}^{2+}$ and HP1043 cannot be phosphorylated in vitro (Fraser et al., 2007; Schar et al., 2005).

Met85 of NblR is at the conserved Thr/Ser position that plays a critical role in activation of RRs. Upon 
(a)

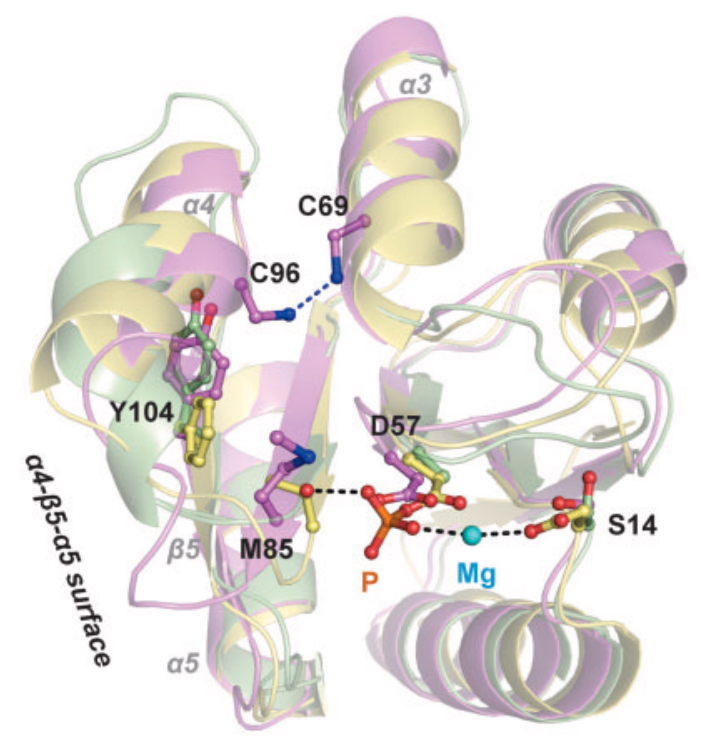

(b)
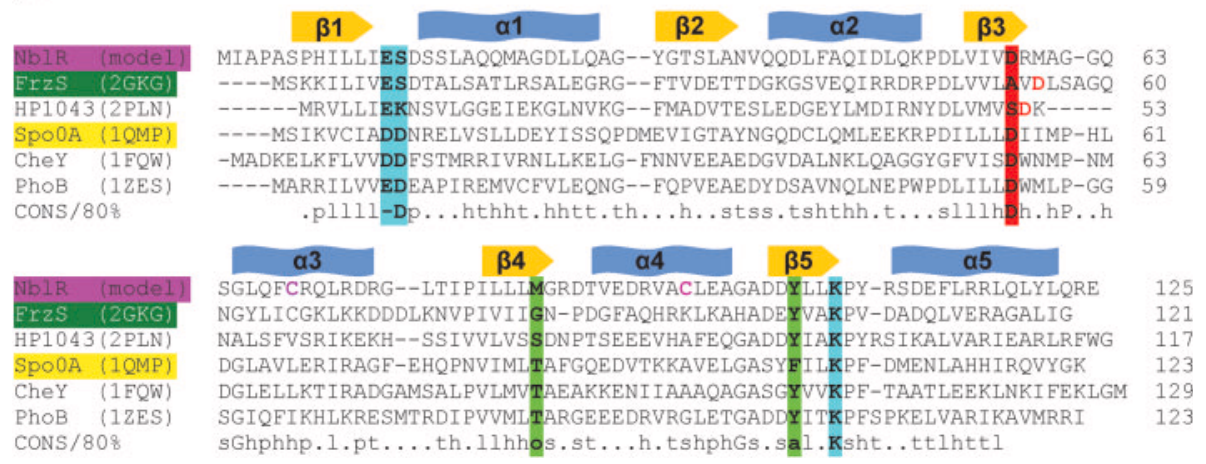

Fig. 1. Structure and sequence comparisons of NbIR RD with canonical and non-canonical RR RDs. (a) Ribbon representation of the 3D model of NbIR RD (magenta) superimposed on the RD structures of the phosphorylated canonical RR Spo0A from $B$. stearothermophilus (yellow; 1QMP) and the non-canonical RR FrzS from M. xanthus (green; 2GkG). The residues at the phosphoaccepting aspartate, the $\mathrm{Mg}^{2+}$-chelating and conformational switch positions are represented in ball-and-stick with carbon atoms in the same colour as the corresponding ribbon structure: oxygen in red, sulphur in blue and phosphorus in orange. Residues are labelled and numbered for NbIR. The two cysteine residues of NbIR are shown as ball-and-stick and the possible disulphide bridge is denoted by a blue broken line. Black broken lines denote contacts with the phosphoryl group and the $\mathrm{Mg}^{2+}$ ion (cyan sphere) in the phosphorylated SpoOA structure. (b) Structure-based sequence alignment for the NbIR model with the isolated RD structures of atypical RRs FrzS (2GKG) from M. xanthus and HP1043 (2PLN) from H. pylori, and canonical RRs SpoOA (1OMP) from B. stearothermophilus, CheY (1FQW) and PhoB (1ZES) from E. coli was performed using LSQKAB (Collaborative Computational Project, Number 4, 1994). Strand and helices of NbIR are indicated above the sequence alignment as labelled yellow and blue boxes, respectively. Consensus sequence for each position ( $>80 \%$ occurrence) was taken from the SMART server (Letunic et al., 2006) and appears below the sequence alignment (CONS/80\% line) printed following the SMART abbreviation code [upper-case letters indicate the corresponding amino acid; lower-case letters correspond to the following amino acid groups: o $(S, T)$, - $(D, E)$, I (I,L,V), a $(F, H, W, Y)$, s $(A, C, D, G, N, P, S, T, V), p$ $(C, D, E, H, K, N, Q, R, S, T)$, t (A,C,D,E,G,H,K,N,Q,R,S,T), h (A,C,F,G,H,I,K,L,M,R,T,V,W,Y), . (any)]. The conserved phosphoaccepting aspartic acid position is shaded in red, the conserved catalytic residue positions in light blue and the switch residue positions in green. The alternative phosphoaccepting aspartate residues in FrzS and HP1043 are highlighted in red and the $\mathrm{NbIR}$ cysteine residues in magenta.

phosphorylation, this conserved Thr/Ser residue is hydrogen bonded with the phosphoryl group, inducing the repositioning of the loop that connects the $\beta 4$ strand with the $\alpha 4$ helix and the concomitant orientation inwards of an exposed aromatic residue in $\beta 5$ (Tyr106 in CheY). This orchestrated movement is called the 'T-loop-Y' coupling switch and its ultimate output is the reorganization of the $\alpha 4-\beta 5-\alpha 5$ surface, leading to changes in the affinity for the 
effector domain, downstream protein targets, or, in the OmpR-PhoB family, receiver dimerization (Dyer \& Dahlquist, 2006). The presence of methionine in this position, which precludes the hydrogen bond with the phosphoryl group, would prevent this activation switch. Thus, the Ser14 and Met85 substitutions would impair phosphorylation and the consequent activation of NblR.

\section{NbIR is not phosphorylated in vitro by acetyl phosphate}

Response regulators can be phosphorylated in vitro and in vivo by the small phosphate donor acetyl phosphate (McCleary \& Stock, 1994). The pta gene, encoding phosphotransacetylase, has not been identified in the genome of Synechococcus and therefore phosphorylation of response regulators by acetyl phosphate is unlikely to be of physiological significance in this cyanobacterium. However, the ability of acetyl phosphate to phosphorylate a given response regulator in vitro would indicate that the protein can be phosphorylated in vivo.

The differences, at the level of primary structure, between the NblR RD and canonical regulators strongly suggested that phosphorylation at the conserved Asp57 is not the mechanism of NblR activation. To investigate this issue, we first performed in vitro phosphorylation assays with acetyl phosphate. Both full-length NblR (6His-NblR) and the NblR RD (6His-NblR-RD) were overproduced and purified. Phosphorylation was compared with RR468, a canonical RR protein from Thermotoga maritima, on a non-denaturing gel (Fig. 2a). Consistent with phosphorylation-induced dimerization in RRs of the $\mathrm{OmpR} / \mathrm{PhoB}$ family (Bachhawat et al., 2005), incubation of RR468 with acetyl phosphate induces a band shift. In contrast,
6His-NblR-RD and 6His-NblR did not change in mobility, suggesting that these proteins were not phosphorylated. Experiments carried out in parallel with NblR and NblR$\mathrm{RD}$ proteins after $6 \mathrm{His}$-tag removal with TEV protease showed equivalent results (data not shown).

To exclude possible artefacts interfering with detection of phosphorylated forms of 6His-NblR-RD and RR468 in native gels, we used additional experimental approaches. Since the addition of a phosphoryl group to proteins reduces their $\mathrm{pI}$ by around 0.2 units (Jeon et al., 2001), we performed 2D gel electrophoresis before and after addition of the phosphodonor. In full agreement with previous results, incubation with acetyl phosphate shifted RR468 towards the acidic part of the gel and had no effect on 6His-NblR-RD (Fig. 2b). Finally, the samples were subjected to mass spectroscopy, confirming that the incubation with acetyl phosphate introduces a single phosphoryl group in RR468 and that 6His-NblR-RD did not change its mass (data not shown), confirming the lack of phosphate incorporation to NblR proteins.

\section{Phosphorylation of Asp57 is not required for NbIR functions}

The structural features of the NblR RD noted above and its lack of phosphorylation by acetyl phosphate strongly suggested that, in spite of conservation, Asp57 was not involved in NblR activation. To determine whether this residue still plays a regulatory role during acclimation to stress, we constructed Synechococcus strains in which Asp57 was replaced by Ala. The strategy for allelic replacement is outlined in Supplementary Fig. S1 (available with the online version of this paper). Strain $\mathrm{NblR}^{\mathrm{D} 57 \mathrm{~A}}-\mathrm{RCS3}$ contains the mutation at its chromosomal emplacement

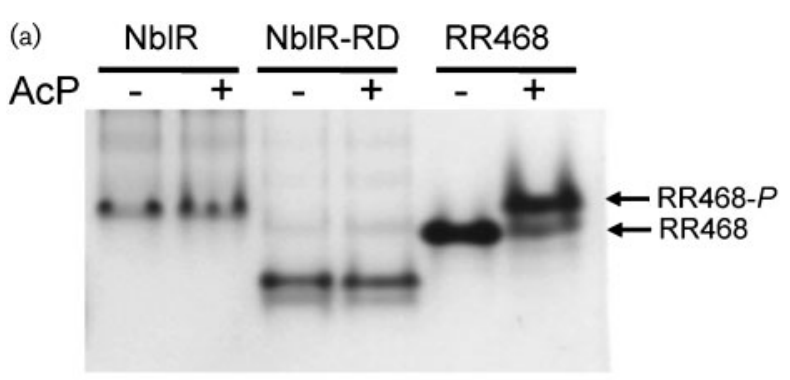

(b)

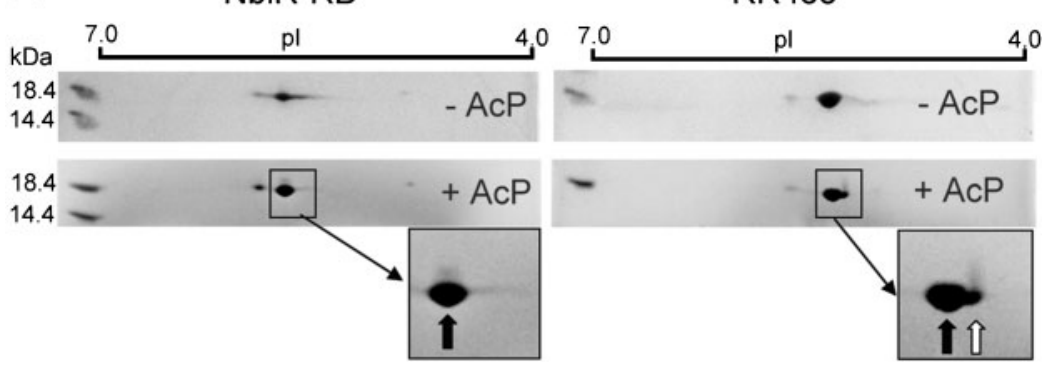

Fig. 2. Phosphorylation with acetyl phosphate and analysis by native and $2 \mathrm{D}$ gel eletrophoresis. (a) 6His-NbIR, 6His-NbIR-RD and RR468 were incubated in the presence $(+\mathrm{AcP})$ or absence $(-\mathrm{AcP})$ of acetyl phosphate and separated according to their charge/mass relation by native PAGE. Unphosphorylated and phosphorylated forms of RR468 are indicated. (b) 6 His-NbIR-RD and RR468 were incubated in presence or absence of acetyl phosphate for $60 \mathrm{~min}$. Samples for 2D gel electrophoresis consisted of the unphosphorylated proteins $(-\mathrm{AcP})$ or a mix of equal amounts of unphosphorylated and phosphorylated proteins $(+A c P)$, where the unphosphorylated protein was used as internal control. The unphosphorylated and phosphorylated forms in + AcP gels are indicated with filled and open arrows, respectively. 
( $n b l R^{\mathrm{D} 57 \mathrm{~A}}$ allele) and the streptomycin-resistance cassette CS3 located downstream. To exclude polar effects and minimize possible artefacts due to the presence of the CS3 cassette, a streptomycin-resistant control strain retaining the wild-type $n b l R$ allele (WT-RCS3) was generated in parallel. Homozygosis for CS3 alleles was promptly achieved and it was confirmed that the presence of the streptomycinresistant cassette CS3 did not confer significant phenotypic differences to the wild-type Synechococcus strain under standard or stress conditions (data not shown). For simplicity, only data produced with the strain WT-RCS3 are shown as control and referred to as wild-type hereafter.

To determine the impact of the D57A substitution on NblR function, we analysed the ability of the $n b l R^{\mathrm{D} 57 \mathrm{~A}}$ mutant to respond to conditions requiring NblR activity. In particular, high light (HL) irradiation and nitrogen deficiency allow clear discrimination between wild-type Synechococcus and $n b l R$ null derivatives (Luque et al., 2001; Salinas et al., 2007; Schwarz \& Grossman, 1998). As shown in Fig. 3(a), when wild-type and mutant derivatives were subjected to HL irradiation only the $n b l R$ null mutant (strain NblR45) ceased growth. The $n b l R^{\mathrm{D} 57 \mathrm{~A}}$ strain continued to grow, at a rate similar to that of the wild-type control. At different times (up to 4 days), drops of all cultures were plated and incubated in various conditions to visually determine their ability to resume growth. As shown in Fig. 3(b), only the null mutant failed to recover appropriately, while the ability of the $n b l R^{\mathrm{D} 57 \mathrm{~A}}$ mutant to recover from stress was similar to that of the wild-type, indicating that Asp57 is not required to increase the resistance of Synechococcus to HL stress (Fig. 3b, panel HL). Equivalent results, i.e. no difference between wild-type and $n b l R^{\mathrm{D} 57 \mathrm{~A}}$ strains in conditions in which the null mutant is clearly impaired, were obtained when cultures were subjected to nitrogen starvation (Fig. 3b, panel $-\mathrm{N}$ ).

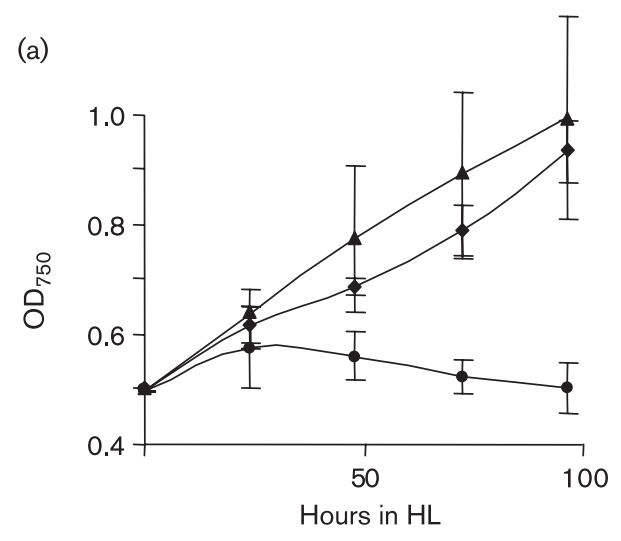

(c)

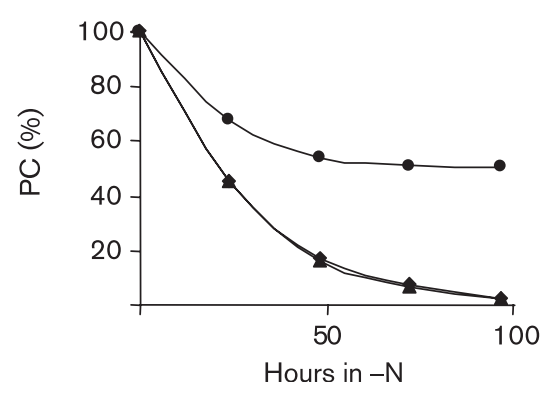

(b)

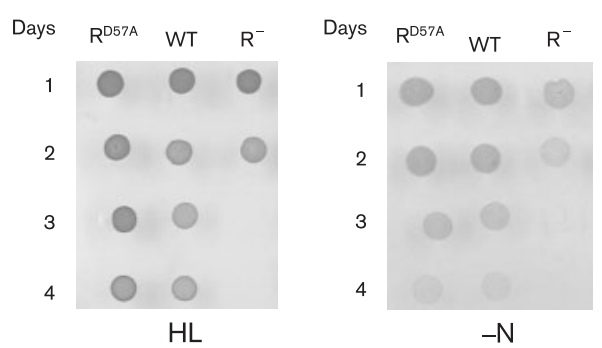

(d)

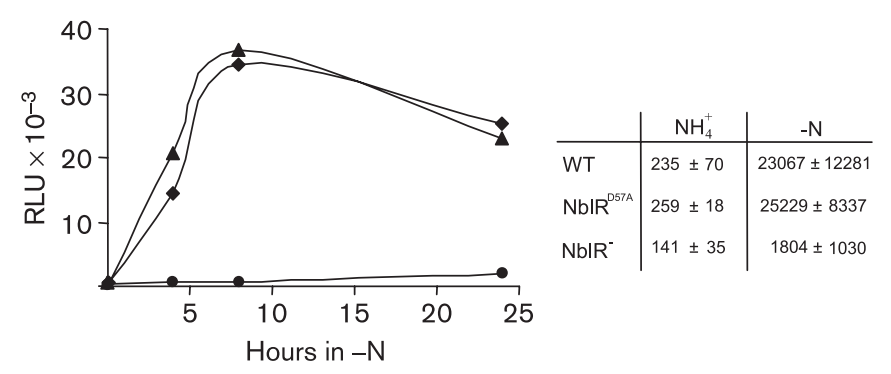

Fig. 3. Effect of the D57A substitution at NbIR on Synechococcus cultures subjected to stress. (a) Growth, as measured by $\mathrm{OD}_{750}$, of WT-RCS3 $(\boldsymbol{\Delta}), \mathrm{NbIR}^{\mathrm{D} 57 \mathrm{~A}}-\mathrm{RCS3}(\boldsymbol{)})$ and NbIR45 $(\bullet)$ at $500 \mu \mathrm{mol}$ photons $\mathrm{m}^{-2} \mathrm{~s}^{-1}$. Mean values and standard deviations of three independent experiments are shown. (b) Drops $(5 \mu \mathrm{l})$ of cultures from NbIR ${ }^{\mathrm{D} 57 \mathrm{~A}}-\mathrm{RCS3}\left(\mathrm{R}^{\mathrm{D} 57 \mathrm{~A}}\right), \mathrm{WT}-\mathrm{RCS3}$ (WT) and NbIR45 $\left(\mathrm{R}^{-}\right)$were spotted on BG11 solid medium after nitrogen deprivation $(-\mathrm{N})$ or high-light $(\mathrm{HL})$ stress conditions up to 4 days, incubated in standard light conditions and photographed 5 days later. A picture taken from one representative experiment is shown in each case. (c) Relative amount of phycocyanin (PC) as a function of time. Strains NbIR ${ }^{\mathrm{D} 57 \mathrm{~A}}-\mathrm{RCS3}$, WTRCS3 and $\mathrm{NbIR}^{-}$were transferred at mid-exponential phase from ammonium-containing to nitrogen-deprived medium ( $\mathrm{N}$ ). Mean values from three or four experiments are shown. (d) Time-course of $\mathrm{P}_{n b / A}$ : : luxAB induction in Synechococcus strains. Cells were grown in the presence of $\mathrm{NH}_{4}^{+}$and when they reached mid-exponential phase, they were shifted to nitrogen-free medium. After nitrogen deprivation at time 0 , bioluminescence from the reporter strains was recorded. Data from one representative experiment out of three is shown. Mean values and standard deviations at time $0\left(\mathrm{NH}_{4}^{+}\right)$and $24 \mathrm{~h}$ after nitrogen deprivation $(-\mathrm{N})$ from three independent experiments are given in the inset table. 
To determine the impact of the D57A substitution on $n b l A$ gene induction and chlorosis, we analysed the absorbance spectra of mutant and control cultures subjected to nitrogen stress as well as the expression of the reporter fusion $\mathrm{P}_{n b l A}:: \operatorname{lux} A B$ (Espinosa et al., 2007), which is positively regulated by NblR (Salinas et al., 2007). As shown in Fig. 3(c), the kinetics of phycocyanin loss were identical in $n b l R^{\mathrm{D} 57 \mathrm{~A}}$ and wild-type cultures subjected to nitrogen starvation, conditions in which the $\mathrm{NblR}^{-}$strain maintained high phycocyanin levels. In line with this, when $n b l R^{\mathrm{D} 57 \mathrm{~A}}$ and wild-type cultures carrying the $\mathrm{P}_{n b l A}:: \operatorname{lu} x A B$ reporter fusion were subjected to nitrogen deprivation, a strong and equal increase in the bioluminiscence signal was observed in wild-type and $n b l R^{\mathrm{D} 57 \mathrm{~A}}$ strains but not in $\mathrm{NblR}^{-}$cultures (Fig. 3d), thus indicating that Asp57 does not play a role in nblA gene activation under the stress conditions used here.

\section{Canonical receivers, pseudo-receivers and the PIARR group}

Several proteins with RD-like structural folds lacking residues involved in aspartic acid phosphorylation and signal transduction and having extended loop regions that align poorly to canonical $\mathrm{RD}$ have been characterized. The cyanobacterial circadian clock protein KaiA (Williams et al., 2002 ) is one of these proteins with a pseudo-RD. However, the RDs of FrzS, HP1043 and NblR are not as divergent from canonical RDs as pseudo-RD (see Fraser et al., 2007 for an extended discussion) and they still contain some of the consensus residues around the canonical phosphorylation pocket and the output face (Fig. 1). Importantly, FrzS, HP1043 and the NblR-modelled RDs maintain the essential nature of the $\alpha 4-\beta 5-\alpha 5$ face, suggesting its involvement in signal propagation. In the case of FrsZ, the switch Tyr and a neighbouring His residue have been shown to be essential for function. Therefore, in spite of the lack of phosphorylation, these atypical RDs conserve key features involved in signal propagation. To recognize the existence of alternative input mechanisms for signal transduction within the two-component RR superfamily, we propose the term PIARR, standing for phosphorylation-independent activation of response regulator. In this manner, in addition to the grouping into classical RR families on the basis of output domain homology, proteins differing in the mechanism of input signalling, the so-called hybrid RDs (Fraser et al., 2007), can also be distinguished on the basis of receiver features.

\section{In vitro and in vivo assays indicate that NbIR is monomeric}

OmpR/PhoB regulators are DNA-binding proteins with a high degree of conservation of the $\alpha 4-\beta 5-\alpha 5$ surface and it has been proposed that they all share a common mechanism of activation that involves dimerization of RDs using the $\alpha 4-\beta 5-\alpha 5$ surface (Gao et al., 2007; ToroRoman et al., 2005). Output domain homology places
NblR and HP1043 with the abundant OmpR/PhoB family of RRs. HP1043 is constitutively active in vivo and purified HP1043 is a dimer whose RD structure resembles the active and phosphorylated form of PhoB. Since helix-turn-helix proteins in general and all characterized $\mathrm{OmpR} / \mathrm{PhoB}$ family members in particular bind to DNA as dimers, it was important to address the oligomerization status of NblR.

To determine the oligomeric state of NblR in vitro, 6His$\mathrm{NblR}$ and 6His-NblR-RD proteins were subjected to gel filtration chromatography on a Superdex 200 column. 6His-NblR and 6His-NblR-RD eluted as single peaks with elution volumes of $15.1 \mathrm{ml}$ and $16.1 \mathrm{ml}$ that corresponded on the calibrated column to masses of 28000 and $16000 \mathrm{Da}$, respectively (Fig. 4a). The calculated masses for the polypeptide of $6 \mathrm{His}-\mathrm{NblR}$ and $6 \mathrm{His}-\mathrm{NblR}-\mathrm{RD}$ are 28590 and $15800 \mathrm{Da}$ respectively, in agreement with a monomeric state. Although our data showed that NblR is not phosphorylated in vitro by small phosphodonors (see above), the effect of the acetyl phosphate in the quaternary structure of NblR was also evaluated. As expected, preincubation with acetyl phosphate had no appreciable effects on the elution volume of either protein (Fig. $4 \mathrm{~b}$ ).

The structural model of NblR strongly suggests the possibility of a disulphide bridge between Cys69 and Cys96 (Fig. 1a). To explore the possibility that the redox state could regulate the $\alpha 4-\beta 5-\alpha 5$ surface and, consequently, dimerization, we also estimated the molecular mass of 6His-NblR and 6His-NblR-RD under oxidizing and reducing conditions. The incubation of both proteins with the reducing agent DTT and subsequent chromatography in the Sephadex 200 column equilibrated with a buffer containing DTT showed that both proteins behaved as monomers (Fig. 4c). To ensure a complete oxidation state, the proteins were incubated with the oxidizing agent $\mathrm{Cu}$-phenanthroline for $30 \mathrm{~min}$ prior to the chromatography. The oxidized proteins showed a similar elution profile to both the non-treated protein and the reduced protein (Fig. 4d). Therefore, in our experimental conditions, the redox state had no major effect on the oligomeric nature of the purified NblR proteins analysed. To assess the impact of the 6His-tag on the quaternary structure, analogous filtration assays were carried out with both proteins after tag removal; these gave similar results (data not shown).

The in vitro data, indicating that $6 \mathrm{His}-\mathrm{NblR}$ and $6 \mathrm{His}-$ NblR-RD are monomeric, are still compatible with NblR activation by dimer formation in Synechococcus and we wondered whether we could find in vivo indications of associations between NblR monomers using two-hybrid interaction assays. We reasoned that, although these systems would not allow specific activation by stress signals, they could be more sensitive than in vitro systems and/or provide a more physiological environment for monomer association. With this in mind, we performed assays using the yeast two-hybrid system, based on 
6His-NbIR

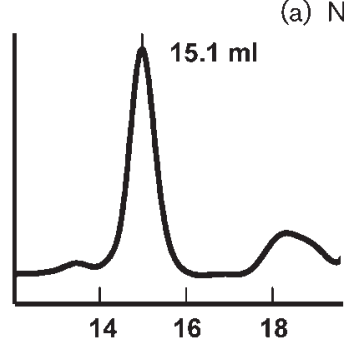

(b) + Acetyl phosphate

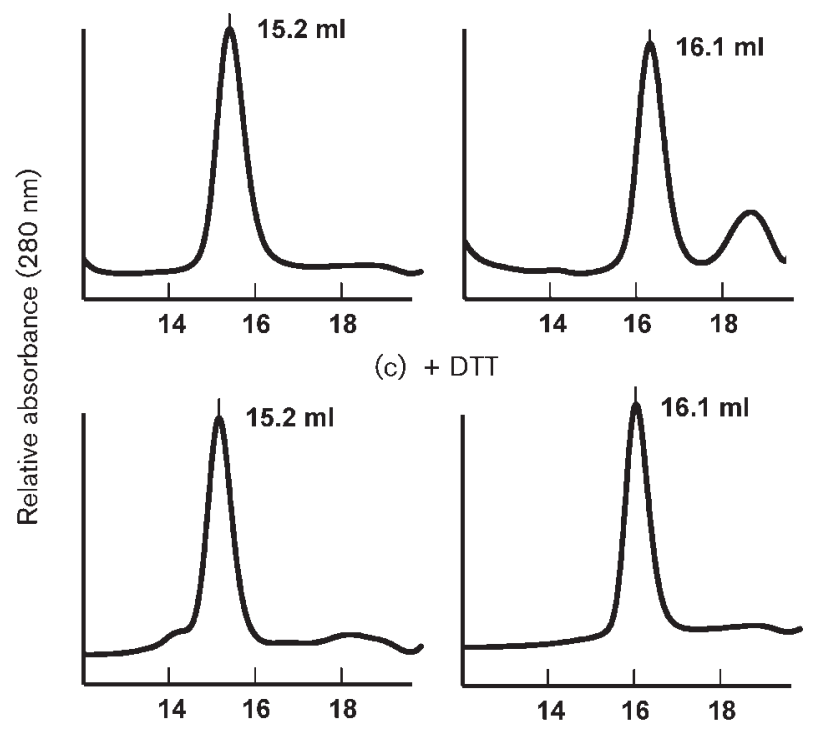

(d) + Cu-phenanthroline

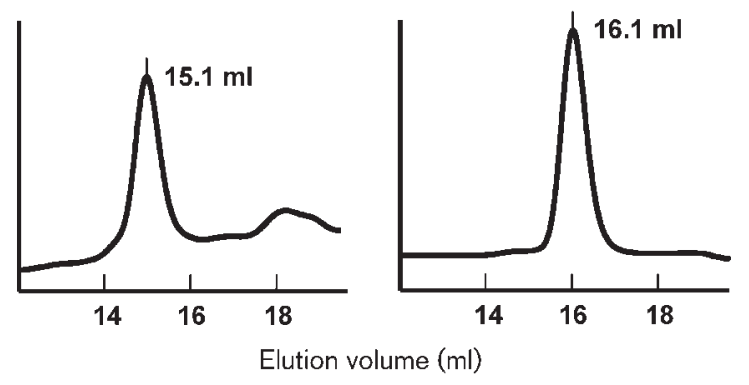

Fig. 4. Gel filtration analysis of $6 \mathrm{His}-\mathrm{NbIR}$ and $6 \mathrm{His}-\mathrm{Nb} / \mathrm{R}-\mathrm{RD}$. The absorbance elution profiles of $6 \mathrm{His}-\mathrm{NbIR}$ (left) and 6HisNbIR-RD (right) proteins after chromatography on Superdex 200 HR under different conditions are shown: (a) native, (b) phosphorylated with acetyl phosphate, (c) reduced with DTT, and (d) oxidized with Cu-phenanthroline. The elution volumes for each peak are indicated.

reconstitution of GAL4 transcriptional activity (Fields \& Song, 1989) and the BACTH system, based on reconstitution of a cyclic AMP signalling cascade in an E. coli cya strain (Karimova et al., 1998). For yeast two-hybrid analyses, constructs included two full-length (NblR) and two C-terminally truncated derivatives of NblR $\left(\mathrm{NblR}^{1-171}\right)$, each one fused to upstream GAL4 domains
(GAL4BD and GAL4AD), and two full-length NblR derivatives, each one fused to C-terminally located GAL4BD and GAL4AD polypeptides (see Table 1 and Fig. 5 for plasmid details). The downstream location of GAL4 domains in fusion proteins was aimed at minimizing possible artefacts resulting from inappropriate conformations of the NblR RD contiguous to the GAL domain, i.e. false negatives. Expression of HIS3, ADE2 and lacZ reporters in Y187/PJ696 diploids containing relevant pairs of fusion proteins was determined as previously described (Burillo et al., 2004). All six protein fusions gave appropriate expression in yeast, but no signal interactions between NblR proteins were found (nine pair combinations of these proteins were tested, Table 3 ). The same result was found with full-length NblR derivatives using the independent BACTH system, thus providing additional evidence of the monomeric conformation of NblR in vivo. Taken together, both in vitro and in vivo analyses indicate that the default state of NblR is monomeric.

\section{Interactions of NbIR with Synechococcus proteins}

The yeast two-hybrid system has been shown to detect specific interactions, between cognate two-component proteins (Martinez-Argudo et al., 2001; Ohta \& Newton, 2003) and between HKs and specific regulators (Espinosa et al., 2006; Martinez-Argudo et al., 2002). In contrast to classical genetic screens, yeast two-hybrid approaches rely on protein-protein interactions, and not on phenotype or viability, a clear advantage when dealing with phenotypes difficult to assay or essential functions. It should be noted that the relatively downstream location of interaction determinants in $\mathrm{HKs}$, usually preceded by $\mathrm{N}$-terminal transmembrane and sensory domains, increases the chances of identifying these proteins as yeast two-hybrid preys. To further increase the chances of detecting interactions of the relevant receiver domain of NblR, we used GAL4BD-NblR and GAL4BD-NblR ${ }^{1-171}$ as baits in Sau3AI and Tsp509Igenerated Synechococcus yeast two-hybrid libraries (Burillo, 2006; Burillo et al., 2004). However, no HK polypeptides were found in the screenings.

The fact that the HK NblS had been identified in $n b l$ screenings, and subsequently proposed as the NblR cognate partner in signal transduction, prompted us to confirm the negative results in our two-hybrid searches and further explore the possibility of a direct protein interaction between NblS and NblR by performing additional and more direct interaction assays with these proteins. To this end, all six GAL4AD or GAL4BD fusions to NblR derivatives used above were assayed in the appropriate combinations with NblS and $\mathrm{NblS}^{272-664}$ (see Table 1 and Fig. 5 for plasmid details). For all ten protein fusions, appropriate expression in yeast was previously verified (Espinosa et al., 2006; and data not shown). In agreement with previous results, analysis of reporter expression in diploids containing relevant pairs of fusion proteins 


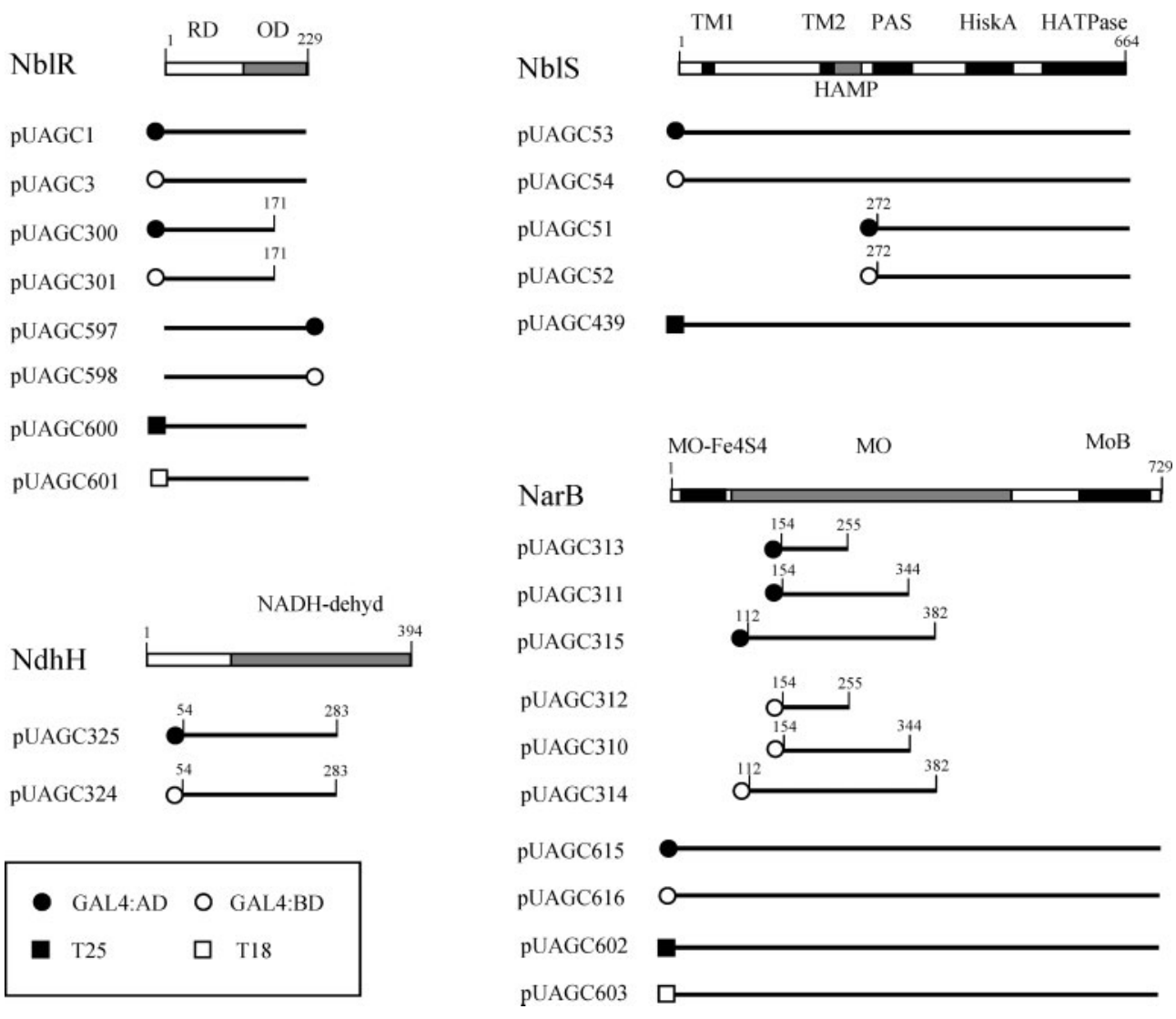

Fig. 5. Schematic representation of the different $\mathrm{NbIR}, \mathrm{NbIS}, \mathrm{NdhH}$ and $\mathrm{NarB}$ polypeptides encoded by two-hybrid plasmids obtained and/or used in this study. Numbers indicate the amino acid boundaries of the polypeptides present in each given plasmid. Domain nomenclature for NbIR and NbIS as in the text. MO-Fe4S4, molybdopterin Fe4S4 domain; MO, molybdopterin domain; MoB, molybdopterin-binding domain; NADH-dehyd, NADH dehydrogenase domain. The position of yeast (GAL4AD and GAL4BD) and bacterial (T25 and T18) domains related to the polypeptides is shown. Symbols used to represent the different GAL4 or CyaA domains are listed in the inset. The short NarB and NdhH polypeptides fused to GAL4AD domains were obtained in yeast two-hybrid screenings using NbIR or $\mathrm{NbIR}^{1-171}$ as bait, respectively.

confirmed lack of interaction between NblS and NblR for all six pairs of fusion proteins tested (Table 3).

The BACTH system provides an independent assay particularly appropriate for membrane-anchored bacterial proteins (Karimova et al., 2005) and might therefore provide a more physiological environment for NblS function. We produced fusion proteins of the two fragments (T25 and T18) of the catalytic domain of Bordetella pertussis adenylate cyclase to NblS, and analysed its ability to complement the $\mathrm{Cya}^{-}$phenotype when paired with NblR. Again, no interaction was found between NblS and NblR derivatives. Since appropriate expression in the host E. coli strain was verified for the T25-NblS protein, but not for the T18-NblS protein, the latter fusion is not included in Table 1 and Fig. 5. Taken together, the yeast and bacterial two-hybrid analyses strongly argue against protein-protein interactions between the two $n b l$ regulators NblS and NblR.

Screening of Synechococcus libraries with NblR polypeptides as baits produced unexpected results. Instead of HK polypeptides, multiple clones containing narB, encoding nitrate reductase, and $n d h H$, encoding a subunit of the $\mathrm{NDH}-1$ complex, were found as preys. In particular, screening of the libraries with GAL4BD-NblR as bait rendered narB preys while $n d h H$ clones appeared in screenings with GAL4BD-NblR ${ }^{1-171}$. From the size of the clones, it can be inferred that $\mathrm{NarB}^{154-255}$, encoding part of the molybdopterin domain, and the fragment $\mathrm{NdhH}^{54-283}$ contain determinants for interaction with NblR. Additional yeast two-hybrid assays validated these interactions and localized the interaction with $\mathrm{NarB}^{154-255}$ to the C-terminal DNA-binding domain of NblR.

The interaction of the output DNA-binding domain of NblR with the nitrate assimilation enzyme was particularly intriguing and prompted us to verify the interaction with the full-length NarB protein and to perform independent protein-protein interactions assays in E. coli. Since appropriate expression of the Synechococcus narB gene leads to nitrate reductase activity in E. coli (Rubio et al., 
Table 3. Yeast two-hybrid interactions

The GAL4AD and GAL4BD fusion proteins carried by diploids are indicated on the left and at the top, respectively. In the case of the NblR fulllength fusions, the position of the GAL4 domain is also indicated. Asterisks refer to absence of proteins fused to GAL4 domains. Levels of interaction were classified according to the strength of the signals, as previously described (Burillo et al., 2004). ND, Not determined.

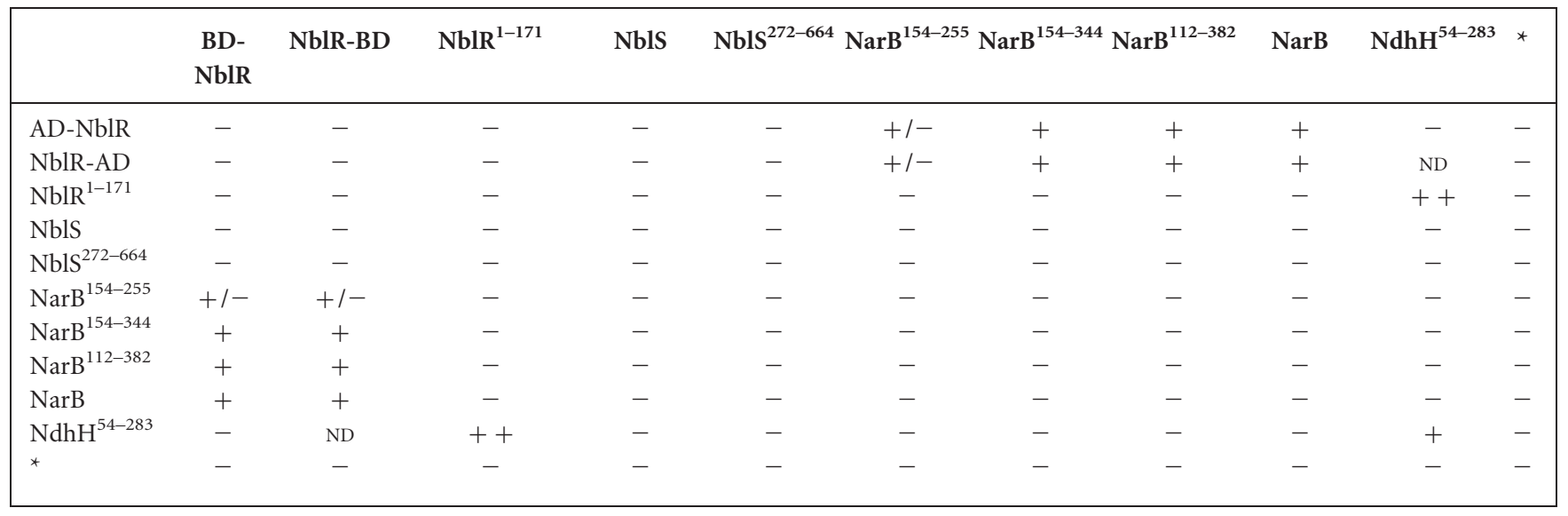

1996), it was important to test the NblR-NarB interaction in this heterologous system. BACTH assays confirmed the interaction between NarB and both NblR and $\mathrm{NblR}^{125-229}$ derivatives, indicating that $\mathrm{NblR}$ and $\mathrm{NarB}$ also have considerable affinity to each other in a prokaryotic intracellular environment.

\section{Integration of stress signals by NbIR and PIARR proteins}

We have shown here that NblR regulation seems to be independent of phosphorylation by HKs and does not fit the two-component paradigm. It should be noted that Kato et al. (2008) have very recently obtained some of the results presented in this work: failure to detect a regulatory phenotype for an independently constructed $n b l R^{\mathrm{D} 57 \mathrm{~A}}$ mutant and detection of specific interactions between NblR and NarB in yeast two-hybrid screenings with NblR. While these authors did not detect $\mathrm{NdhH}$ polypeptides as preys, they detected a protein not found by us, MreC. Fishing of different preys is not surprising given that the strategies for library construction differed between the two laboratories. The challenge is now to determine the physiological significance of the protein-protein interactions with NblR detected by yeast two-hybrid methods.

Nitrogen and sulphur starvation stress signals did not regulate $n b l R$ transcripts and purified NblR had very low affinity for its target regulatory region at $n b l A$ (Luque et al., 2001), suggesting that, as expected for a RR, the regulation is at the level of protein activitiy. NblR might be regulated as other $\mathrm{OmpR} / \mathrm{PhoB}$ proteins, being able to switch between active dimers and inactive monomers. If that is the case, the implication is that phosphorylation would not be the only means to achieve activation by dimerization within the OmpR/PhoB family.

It is now clear that phosphorylation of canonical RD, the paradigm for signal input into RRs, must coexist with alternative ways of communication operating in PIARR proteins. It can be anticipated that research with these atypical RRs would greatly deepen our understanding of prokaryotic signal transduction.

\section{ACKNOWLEDGEMENTS}

We thank S. Burillo, I. Fuentes, K. Lauchoubi and J. Espinosa for yeast two-hybrid work and/or plasmid construction, J. J. Calvete for mass spectroscopy analyses, and R. Dixon, K. Forchhammer and J. Espinosa for constructive discussions. This work was supported by the Ministerio de Educación y Ciencia (grants BFU2006-12424 to A.C. and BIO2005-00153 to A.M.) and the Generalitat Valenciana (grant ACOMP06/083 to A.C.). M. L. L.-R. is a fellow of the Fundación Mutua Madrileña Automovilística.

\section{REFERENCES}

Ausubel, F. M., Brent, R., Kingston, R. E., Moore, D. D., Seidman, J. J., Smith, J. A. \& Struhl, K. (editors) (1999). Short Protocols in Molecular Biology: a Compendium of Methods from Current Protocols in Molecular Biology. New York: Wiley.

Bachhawat, P., Swapna, G. V., Montelione, G. T. \& Stock, A. M. (2005). Mechanism of activation for transcription factor PhoB suggested by different modes of dimerization in the inactive and active states. Structure 13, 1353-1363.

Bartel, P., Chien, C. T., Sternglanz, R. \& Fields, S. (1993). Elimination of false positives that arise in using the two-hybrid system. Biotechniques 14, 920-924.

Brunger, A. T., Adams, P. D., Clore, G. M., DeLano, W. L., Gros, P., Grosse-Kunstleve, R. W., Jiang, J. S., Kuszewski, J., Nilges, M. \& other authors (1998). Crystallography \& NMR system: a new software suite for macromolecular structure determination. Acta Crystallogr D Biol Crystallogr 54, 905-921.

Burillo, S. (2006). Identificación y caracterización de componentes celulares implicados en transducción de señales en Synechococcus sp. PCC 7942. PhD thesis, Universidad de Alicante, Spain.

Burillo, S., Luque, I., Fuentes, I. \& Contreras, A. (2004). Interactions between the nitrogen signal transduction protein PII and $\mathrm{N}$ - 
acetylglutamate kinase in organisms that perform oxygenic photosynthesis. J Bacteriol 186, 3346-3354.

Casino, P., Fernandez-Alvarez, A., Alfonso, C., Rivas, G. \& Marina, A. (2007). Identification of a novel two component system in Thermotoga maritima. Complex stoichiometry and crystallization. Biochim Biophys Acta 1774, 603-609.

Collaborative Computational Project, Number 4 (1994). The CCP4 suite: programs for protein crystallography. Acta Crystallogr D Biol Crystallogr 50, 760-763.

Collier, J. L. \& Grossman, A. R. (1992). Chlorosis induced by nutrient deprivation in Synechococcus sp. strain PCC 7942: not all bleaching is the same. J Bacteriol 174, 4718-4726.

Collier, J. L. \& Grossman, A. R. (1994). A small polypeptide triggers complete degradation of light-harvesting phycobiliproteins in nutrient-deprived cyanobacteria. EMBO J 13, 1039-1047.

Dyer, C. M. \& Dahlquist, F. W. (2006). Switched or not?: the structure of unphosphorylated CheY bound to the $\mathrm{N}$ terminus of FliM. J Bacteriol 188, 7354-7363.

Espinosa, J., Fuentes, I., Burillo, S., Rodriguez-Mateos, F. \& Contreras, A. (2006). SipA, a novel type of protein from Synechococcus sp. PCC 7942, binds to the kinase domain of NblS. FEMS Microbiol Lett 254, 41-47.

Espinosa, J., Forchhammer, K. \& Contreras, A. (2007). Role of the Synechococcus PCC 7942 nitrogen regulator protein PipX in NtcAcontrolled processes. Microbiology 153, 711-718.

Fields, S. \& Song, O. (1989). A novel genetic system to detect proteinprotein interactions. Nature 340, 245-246.

Fraser, J. S., Merlie, J. P., Jr, Echols, N., Weisfield, S. R., Mignot, T., Wemmer, D. E., Zusman, D. R. \& Alber, T. (2007). An atypical receiver domain controls the dynamic polar localization of the Myxococcus xanthus social motility protein FrzS. Mol Microbiol 65, 319-332.

Gao, R., Mack, T. R. \& Stock, A. M. (2007). Bacterial response regulators: versatile regulatory strategies from common domains. Trends Biochem Sci 32, 225-234.

Golden, S. S. \& Sherman, L. A. (1984). Optimal conditions for genetic transformation of the cyanobacterium Anacystis nidulans R2. J Bacteriol 158, 36-42.

Grossman, A. R., Schaefer, M. R., Chiang, G. G. \& Collier, J. L. (1993). The phycobilisome, a light-harvesting complex responsive to environmental conditions. Microbiol Rev 57, 725-749.

Grossman, A. R., Bhaya, D. \& He, Q. (2001). Tracking the light environment by cyanobacteria and the dynamic nature of light harvesting. J Biol Chem 276, 11449-11452.

Hanahan, D. (1985). Techniques for transformation of Escherichia coli. In DNA Cloning, pp. 109-135. Edited by D. M. Glover. Oxford, UK: IRL Press.

Harper, J. W., Adami, G. R., Wei, N., Keyomarsi, K. \& Elledge, S. J. (1993). The p21 Cdk-interacting protein Cip1 is a potent inhibitor of G1 cyclin-dependent kinases. Cell 75, 805-816.

Hubbard, J. A., MacLachlan, L. K., King, G. W., Jones, J. J. \& Fosberry, A. P. (2003). Nuclear magnetic resonance spectroscopy reveals the functional state of the signalling protein CheY in vivo in Escherichia coli. Mol Microbiol 49, 1191-1200.

James, P., Halladay, J. \& Craig, E. A. (1996). Genomic libraries and a host strain designed for highly efficient two-hybrid selection in yeast. Genetics 144, 1425-1436.

Jeon, Y., Lee, Y. S., Han, J. S., Kim, J. B. \& Hwang, D. S. (2001). Multimerization of phosphorylated and non-phosphorylated ArcA is necessary for the response regulator function of the Arc twocomponent signal transduction system. J Biol Chem 276, 4087340879.
Kappell, A. D., Bhaya, D. \& van Waasbergen, L. G. (2006). Negative control of the high light-inducible hliA gene and implications for the activities of the NblS sensor kinase in the cyanobacterium Synechococcus elongatus strain PCC 7942. Arch Microbiol 186, 403-413.

Karimova, G., Pidoux, J., Ullmann, A. \& Ladant, D. (1998). A bacterial two-hybrid system based on a reconstituted signal transduction pathway. Proc Natl Acad Sci U S A 95, 5752-5756.

Karimova, G., Dautin, N. \& Ladant, D. (2005). Interaction network among Escherichia coli membrane proteins involved in cell division as revealed by bacterial two-hybrid analysis. J Bacteriol 187, 2233-2243.

Kato, H., Chibazakura, T. \& Yoshikawa, H. (2008). NblR is a novel one-component response regulator in the cyanobacterium Synechococcus elongatus PCC 7942. Biosci Biotechnol Biochem 72, 1072-1079.

Kopp, J. \& Schwede, T. (2004). The SWISS-MODEL repository of annotated three-dimensional protein structure homology models. Nucleic Acids Res 32, D230-D234.

Lee, G. F., Lebert, M. R., Lilly, A. A. \& Hazelbauer, G. L. (1995). Transmembrane signaling characterized in bacterial chemoreceptors by using sulfhydryl cross-linking in vivo. Proc Natl Acad Sci U S A 92, 3391-3395.

Lee, S. Y., Cho, H. S., Pelton, J. G., Yan, D., Berry, E. A. \& Wemmer, D. E. (2001). Crystal structure of activated CheY. Comparison with other activated receiver domains. J Biol Chem 276, 16425-16431.

Letunic, I., Copley, R. R., Pils, B., Pinkert, S., Schultz, J. \& Bork, P. (2006). SMART 5: domains in the context of genomes and networks. Nucleic Acids Res 34, D257-D260.

Lewis, R. J., Brannigan, J. A., Muchova, K., Barak, I. \& Wilkinson, A. J. (1999). Phosphorylated aspartate in the structure of a response regulator protein. J Mol Biol 294, 9-15.

Luque, I., Zabulon, G., Contreras, A. \& Houmard, J. (2001). Convergence of two global transcriptional regulators on nitrogen induction of the stress-acclimation gene $n b l A$ in the cyanobacterium Synechococcus sp. PCC 7942. Mol Microbiol 41, 937-947.

Martinez-Argudo, I., Martin-Nieto, J., Salinas, P., Maldonado, R., Drummond, M. \& Contreras, A. (2001). Two-hybrid analysis of domain interactions involving $\mathrm{NtrB}$ and $\mathrm{NtrC}$ two-component regulators. Mol Microbiol 40, 169-178.

Martinez-Argudo, I., Salinas, P., Maldonado, R. \& Contreras, A. (2002). Domain interactions on the $n t r$ signal transduction pathway: two-hybrid analysis of mutant and truncated derivatives of histidine kinase NtrB. J Bacteriol 184, 200-206.

McCleary, W. R. \& Stock, J. B. (1994). Acetyl phosphate and the activation of two-component response regulators. J Biol Chem 269, 31567-31572.

Millson, S. H., Truman, A. W. \& Piper, P. W. (2003). Vectors for N- or C-terminal positioning of the yeast Gal4p DNA binding or activator domains. Biotechniques 35, 60-64.

Morris, A. L., MacArthur, M. W., Hutchinson, E. G. \& Thornton, J. M. (1992). Stereochemical quality of protein structure coordinates. Proteins 12, 345-364.

Myers, J., Graham, J. R. \& Wang, R. T. (1980). Light harvesting in Anacystis nidulans studied in pigment mutants. Plant Physiol 66, 1144-1149.

Ohta, N. \& Newton, A. (2003). The core dimerization domains of histidine kinases contain recognition specificity for the cognate response regulator. J Bacteriol 185, 4424-4431.

Pieper, U., Eswar, N., Braberg, H., Madhusudhan, M. S., Davis, F. P., Stuart, A. C., Mirkovic, N., Rossi, A., Marti-Renom, M. A. \& other authors (2004). MODBASE, a database of annotated comparative 
protein structure models, and associated resources. Nucleic Acids Res 32, D217-D222.

Roder, K. H., Wolf, S. S. \& Schweizer, M. (1996). Refinement of vectors for use in the yeast two-hybrid system. Anal Biochem 241, 260-262.

Rubio, L. M., Herrero, A. \& Flores, E. (1996). A cyanobacterial narB gene encodes a ferredoxin-dependent nitrate reductase. Plant Mol Biol 30, 845-850.

Salinas, P., Ruiz, D., Cantos, R., Lopez-Redondo, M. L., Marina, A. \& Contreras, A. (2007). The regulatory factor SipA provides a link between $\mathrm{NblS}$ and NblR signal transduction pathways in the cyanobacterium Synechococcus sp. PCC 7942. Mol Microbiol 66, 1607-1619.

Schar, J., Sickmann, A. \& Beier, D. (2005). Phosphorylationindependent activity of atypical response regulators of Helicobacter pylori. J Bacteriol 187, 3100-3109.

Schwarz, R. \& Grossman, A. R. (1998). A response regulator of cyanobacteria integrates diverse environmental signals and is critical for survival under extreme conditions. Proc Natl Acad Sci U S A 95, 11008-11013.

Sendersky, E., Lahmi, R., Shaltiel, J., Perelman, A. \& Schwarz, R. (2005). NblC, a novel component required for pigment degradation during starvation in Synechococcus PCC 7942. Mol Microbiol 58, 659-668.

Stock, A. M., Robinson, V. L. \& Goudreau, P. N. (2000). Twocomponent signal transduction. Annu Rev Biochem 69, 183-215.

Studier, F. W. (2005). Protein production by auto-induction in high density shaking cultures. Protein Expr Purif 41, 207-234.

Toro-Roman, A., Mack, T. R. \& Stock, A. M. (2005). Structural analysis and solution studies of the activated regulatory domain of the response regulator ArcA: a symmetric dimer mediated by the $\alpha 4-\beta 5$ a5 face. J Mol Biol 349, 11-26.

van Waasbergen, L. G., Dolganov, N. \& Grossman, A. R. (2002). nblS, a gene involved in controlling photosynthesis-related gene expression during high light and nutrient stress in Synechococcus elongatus PCC 7942. J Bacteriol 184, 2481-2490.

Williams, S. B., Vakonakis, I., Golden, S. S. \& LiWang, A. C. (2002). Structure and function from the circadian clock protein KaiA of Synechococcus elongatus: a potential clock input mechanism. Proc Natl Acad Sci U S A 99, 15357-15362.

Edited by: A. Wilde 